\title{
Effect of ascending, descending, and irregular order of varied reward magnitude'
}

\author{
JAMES BOWEN, University of Texas at Arlington, Arlington, \\ Texas 76010
}

Three groups of 12 rats each were trained in a straight alley nunway. All Ss received 3 massed trials and 3 different magnitudes of reward each day for 24 days. Group $A$ received the rewards in ascending order of magnitude, Group $D$ in descending order, and Group $C$ in irregular order. Following acquisition training, each $S$ received three massed extinction trials per day for eight days. All three groups reached the same acquisition asymptote at the same rate after which Group $D$ showed a significant decremental trend such that Groups $A$ and $C$ were running faster than $D$ at the end of acquisition. No group showed any evidence of pattern learning. Group $D$ also extinguished at a faster rate than Groups $A$ and $C$ which did not differ.

This experiment was a test of a prediction derived from Capaldi's (1967) sequential hypothesis of instrumental learning. The primary assumption made by Capaldi is that instrumental performance is regulated mainly by distinctive internal stimuli generated by differential reward conditions. A further assumption is that these internal stimuli may be represented by a similarity dimension such that there is more stimulus generalization to the stimulus aftereffect of nonreward ( $\mathrm{S}^{N}$ ) from the aftereffect of a small reward $\left(S^{S}\right)$ than from the aftereffect of a large reward $\left(S^{L}\right)$. It follows from these assumptions that if during reinforced responding, the reward magnitude is shifted progressively upward, resistance to extinction should be greater than if reward magnitude is shifted progressively downward. If the rewards are given in irregular order of magnitude, and a reasonably long acquisition series is given, resistance to extinction should be at least as great as when given in ascending order, and perhaps slightly more so. Consider, for example, three reward magnitudes (small, medium, and large) given to $\mathrm{Ss}$ in either ascending, descending, or irregular order daily over a period of several days. For the ascending group, $S^{S}$ and $S^{M}$ would become conditioned to the instrumental response because each is present on a rewarded trial, but not $S^{L}$ since the large reward always occurs last. For the descending group, $S^{L}$ and $S^{M}$ would become conditioned, but not $\mathrm{S}^{\mathrm{S}}$. For the irregular group, all three cues would become conditioned since none of the three reward magnitudes always occurs last. Thus, given enough training, the irregular group should be equal or more resistant to extinction than the ascending group, depending upon whether a significant amount of stimulus generalization to $S^{N}$ from $S^{L}$ occurs. Both should be more resistant than the descending group since there should be more generalization to $S^{N}$ from $S^{S}$ than from $S^{L}$. Figure 1 illustrates the pertinent conditions involved in making the predictions.

\section{METHOD}

The Ss were 36 experimentally naive male hooded rats selected from the colony maintained by the University of Texas at Arlington. The Ss were about 90 days old at the beginning of training.

The apparatus was a Hunter standard Model 360 small animal runway. In brief, it was a straight alley 33 in. in overall length. Running speed over the middle $18 \mathrm{in.}$ of the alley was timed on each trial by means of a Standard Electric Timer which was started and stopped by photocells.

Pretraining. The Ss were fed wet mash for $1 \mathrm{~h}$ in their home cages each day following handling or training. For the first 10 days, Ss were handled in groups of nine for $30 \mathrm{~min}$ each. On the eleventh day Ss explored the alley in groups of four for $10 \mathrm{~min}$ each, and on the twelf th day Ss were placed directly into the goal box and allowed to eat two $97 \mathrm{mg}$ Noyes pellets. Each $\mathrm{S}$ was then randomly assigned to one of three groups with the aid of a random numbers table. Day 1 of acquisition training began the following day.
Acquisition training. Each $\mathrm{S}$ was given three massed trials per day for 24 days. For Group A, one $97 \mathrm{mg}$ Noyes pellet was the reward for the first trial, four for the second, and eight for the third. For Group D, the rewards were 8, 4, and 1 pellets, respectively. For Group $I$, the rewards were given in 12 randomized blocks of the six possible orders. The Ss were run in blocks of three, one $S$ from each group, with the order of each $S$ randomly determined. A $15-\mathrm{sec}$ intertrial interval was used for each S. Two Ss in Group D became ill and were dropped from the experiment on Day 18. All their times were disregarded.

Extinction. Each $\mathrm{S}$ was given three nonrewarded trials per day for four days. The $S$ was confined in the goal box for $15 \mathrm{sec}$ on each trial unless it failed to enter within $60 \mathrm{sec}$ of being placed in the start box. After $60 \mathrm{sec}$ it was removed directly from the alley. RESULTS

All analyses were done on the logarithms of the running times. The main experimental findings are presented in Fig. 2.

Acquisition. There were no significant group differences over the first six days, but by the last two days Group D was running significantly slower than Group A and I which did not differ (for Groups, $F(2,31)=3.48, p<.05$; for $A-I$, vs $D, F(1,31)=4.47$, $p<.05$; for $A$ vs $I, F(1,31)=1.21)$. There was no evidence for pattern learning (for Reward Magnitude, $F(2,62)=.82$ ), nor was there a Magnitude by Groups interaction $(F(4,62)=1.56)$ Since a plot of acquisition performance indicated a gradual slowing trend on the part of Group D after Trial 18, a trend analysis using blocks of nine trials over Trials 19 through 72 was done. There was a significant decremental linear trend for Group D $(F(1,217)=12.1$, $\mathrm{p}<.001)$ but no significant linear trend effect for either Group $A$ $(\mathrm{F}(1,217)<1)$ or Group I $(\mathrm{F}(1,217)<1)$.

Extinction. Since there were significant group acquisition differences, a repeated measures analysis over terminal acquisition level (TAL), i.e., mean of the last six acquisition trials, and mean extinction time (MET) was done. The Groups $(\mathrm{F}(2,31)=7.25$, $p<.01)$, Phase $(F(1,31)=94.00, p<.001)$, and Groups by Phase $(F(2,31)=4.63)$ effects were significant. Orthogonal comparisons were done on both the Groups and Groups by Phase effects. A comparison of Groups $A$ and $I$ vs $D$ was significant $(F(1,31)=10.1, p<.01)$ but a comparison of Group A vs I was not $(F(1,31)=.89)$. The interaction of Group $A$ and $I$ vs $D$ over phases was significant $(F(1,31)=16.62, p<.01)$, but the interaction of Group A vs Group I was not $(F(1,31)=1.21)$. These results should be interpreted to mean that Group D extinguished at a faster rate than Groups A and I which did not differ.
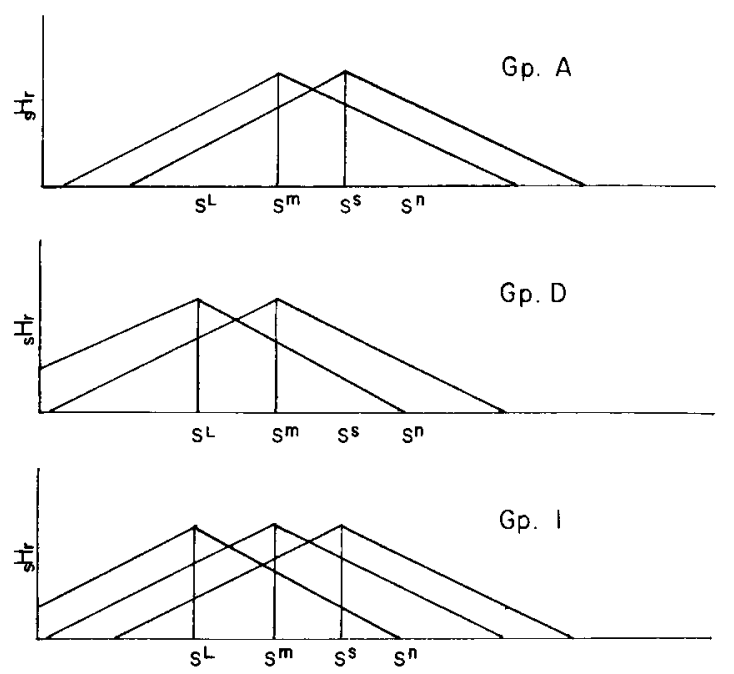

Fig. 1. Generalization from $S^{L}, S^{M}$, and $S^{S}$ to $S^{N}$. 


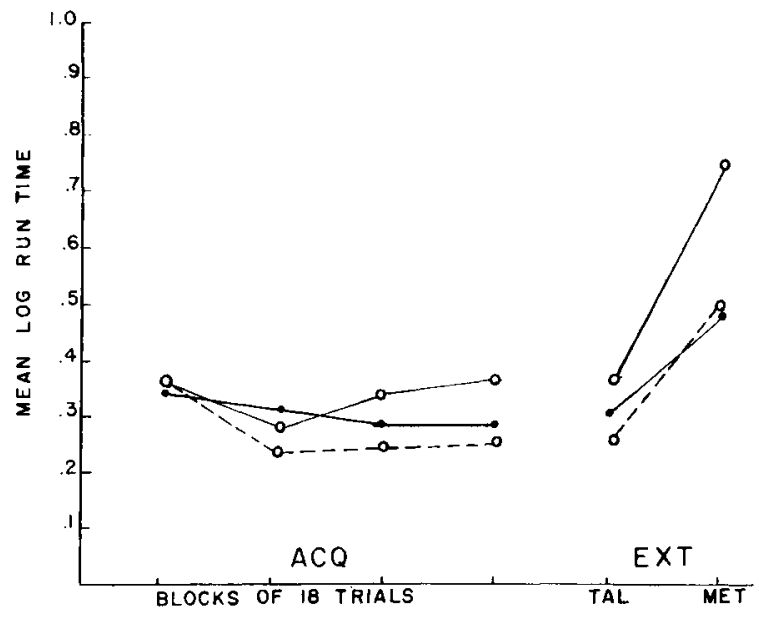

Fig. 2. Mean log run times in acquisition and extinction.

\section{DISCUSSION}

The experimental hypotheses were concerned with extinction performance and were confirmed. Groups $\mathbf{A}$ and I were more resistant to extinction than Group D.
Even though the primary dependent variables for predictions from Capaldi's sequential effects model are measures of extinction performance, the acquisition results are certainly consistent with a general sequential effects approach. For if only intratrial effects were operating, no acquisition differences should have obtained. A tentative interpretation for the acquisition difference is that after a number of rewarded trials the transition from one incentive magnitude to a smaller incentive generates inhibition. Thus, for Group D, 48 of the 72 acquisition trials involve transitions to smaller incentives compared with 24 for Group I and one for Group A. It follows, of course, that after enough training Group 1 should also be less resistant to extinction than Group A.

The term "incentive" seems more appropriate than reward magnitude, since Jensen \& Rey (1968) observed a similar effect when manipulating the amount of reward seen by Ss while holding constant the amount actually consumed. REFERENCES

CAPALDI, E. J. A sequential hypothesis of instrumental learning. In K. W. Spence and J. T. Spence (Eds.), Psychology of learning and motivation. New York: Academic Press, Inc., 1967.

JENSEN, G. D., \& REY, R. P. Runway performance under "hom of plenty" conditions versus gradual diminution of reward supply. Joumal of Experimental Psychology, 1968, 76, 7.11.

NOTE

1. This investigation was supported in part by Public Health Service Research Grant MH 11694-01 from the National Institute of Mental Health. 